\title{
SARS-CoV-2 specific T cells induced by both SARS- CoV-2 infection and mRNA vaccination broadly cross-recognize omicron
}

Julia Lang-Meli

University of Freiburg

Hendrik Luxenburger

University of Freiburg

Katharina Wild

University of Freiburg

Vivien Karl

University of Freiburg

Valerie Oberhardt

University of Freiburg

Elahe Salimi Alizei

University of Freiburg

Anne Graeser

University of Freiburg

Matthias Reinscheid

University of Freiburg

Natascha Roehlen

University of Freiburg

Sebastian Giese

University of Freiburg

Kevin Ciminski

University of Freiburg

Veronika Götz

University of Freiburg

Dietrich August

University of Freiburg

Siegbert Rieg

University of Freiburg

Cornelius Waller

University of Freiburg

Tobias Wengenmayer https://orcid.org/0000-0001-5397-7497 
University of Freiburg

\section{Dawid Staudacher}

University of Freiburg

\section{Bertram Bengsch}

University of Freiburg

\section{Georg Kochs}

Freiburg University Medical Center https://orcid.org/0000-0003-0187-559X

\section{Martin Schwemmle}

University Medical Center Freiburg https://orcid.org/0000-0002-2972-6855

\section{Florian Emmerich}

University of Freiburg

\section{Tobias Boettler}

Department of Medicine II, Medical Center - University of Freiburg, Germany https://orcid.org/00000002-1195-055X

\section{Robert Thimme}

University Hospital Freiburg

\section{Maike Hofmann ( $\checkmark$ maike.hofmann@uniklinik-freiburg.de )}

University of Freiburg

\section{Christoph Neumann-Haefelin}

Department of Medicine II (Gastroenterology, Hepatology, Endocrinology and Infectious Diseases), Freiburg University Medical Center, Faculty of Medicine, University of Freiburg, Freiburg, Germany https://orcid.org/0000-0001-7351-1387

\section{Brief Communication}

Keywords: T cells, SARS-CoV-2, mRNA vaccination, COVID-19, Omicron, B.1.1.529

Posted Date: January 21st, 2022

DOl: https://doi.org/10.21203/rs.3.rs-1269004/v1

License: (c) (i) This work is licensed under a Creative Commons Attribution 4.0 International License. Read Full License 


\section{SARS-CoV-2 specific $T$ cells induced by both SARS-CoV-2 infection and mRNA} vaccination broadly cross-recognize omicron

Julia Lang-Meli ${ }^{1,2 *}$, Hendrik Luxenburger ${ }^{1,2 *}$, Katharina Wild ${ }^{1,3 *}$, Vivien Karl ${ }^{1,4 *}$, Valerie Oberhardt $^{1,4 *}$, Elahe Salimi Alizei ${ }^{1,3}$, Anne Graeser ${ }^{1}$, Matthias Reinscheid ${ }^{1,4}$, Natascha Roehlen ${ }^{1}$, Sebastian Giese ${ }^{5}$, Kevin Ciminski ${ }^{5}$, Veronika Götz ${ }^{1}$, Dietrich August ${ }^{1}$, Siegbert Rieg $^{1}$, Cornelius F. Waller ${ }^{6}$, Tobias Wengenmayer ${ }^{7,8}$, Dawid Staudacher ${ }^{7,8}$, Bertram Bengsch $^{1,9}$, Georg Kochs ${ }^{5}$, Martin Schwemmle ${ }^{5}$, Florian Emmerich ${ }^{10}$, Tobias Boettler,11\#, Robert Thimme ${ }^{1 \# \S}$, Maike Hofmann ${ }^{1 \# \S}$, Christoph Neumann-Haefelin ${ }^{1 \# \S}$

${ }^{1}$ Department of Medicine II (Gastroenterology, Hepatology, Endocrinology and Infectious Diseases), Freiburg University Medical Center, Faculty of Medicine, University of Freiburg, Freiburg, Germany

${ }^{2} \mathrm{MMM}$-PACT, Faculty of Medicine, University of Freiburg, Freiburg, Germany

${ }^{3}$ Faculty of Chemistry and Pharmacy, University of Freiburg, Freiburg, Germany

${ }^{4}$ Faculty of Biology, University of Freiburg, Freiburg, Germany

${ }^{5}$ Institute of Virology, Freiburg University Medical Center, Faculty of Medicine, University of Freiburg, Freiburg, Germany

${ }^{6}$ Department of Haematology, Oncology \& Stem Cell Transplantation, Freiburg University Medical Center, Faculty of Medicine, University of Freiburg

${ }^{7}$ Department of Medicine III (Interdisciplinary Medical Intensive Care), Freiburg University Medical Center, Faculty of Medicine, University of Freiburg, Freiburg, Germany

${ }^{8}$ Department of Cardiology and Angiology I, Heart Center, Freiburg University Medical Center, Faculty of Medicine, University of Freiburg, Freiburg, Germany

${ }^{9}$ Signalling Research Centres BIOSS and CIBSS, University of Freiburg, Freiburg, Germany ${ }^{10}$ Institute for Transfusion Medicine and Gene Therapy, Freiburg University Medical Center, Faculty of Medicine, University of Freiburg, Germany

${ }^{11}$ Berta-Ottenstein Programme, Faculty of Medicine, University of Freiburg

*equally contributing first authors; \#equally contributing last authors; ${ }^{\S}$ corresponding authors

Materials and Correspondence:

Christoph Neumann-Haefelin: christoph.neumann-haefelin@uniklinik-freiburg.de

Maike Hofmann: maike.hofmann@uniklinik-freiburg.de

Robert Thimme: robert.thimme@uniklinik-freiburg.de

Hugstetter Straße 55, 79106 Freiburg, Germany 


\section{Abstract}

The SARS-CoV-2 variant of concern (VOC) omicron (B1.1.529) is associated with high infectivity and efficient evasion from humoral immunity induced by previous infection or vaccination. In omicron-infected individuals who have been vaccinated or infected before, severe disease seems to be relatively infrequent pointing towards protection by previously primed SARS-CoV-2-specific $T$ cells that cross-recognize omicron. By performing a comprehensive in-depth comparison of the SARS-CoV-2-specific T cell epitope repertoire after natural infection versus after mRNA vaccination, we here demonstrate that spike-derived epitopes are not dominantly targeted in convalescents compared to non-spike epitopes. In vaccinees, however, we detected a broader spike-specific $\mathrm{T}$ cell response compared to convalescents reflected by a more diverse repertoire of dominantly targeted spike-specific $T$ cell epitopes. Booster mRNA vaccination induced a broader spike-specific $T$ cell response in convalescents but not in vaccinees with complete initial vaccination. In convalescents and vaccinees, the targeted $T$ cell epitopes are broadly conserved between ancestral and omicron SARS-CoV-2 variants. Hence, our data emphasize the relevance of mRNA vaccine-induced spike-specific CD8+ $T$ cell responses in combating emerging SARS-CoV-2 VOC including omicron and support the benefit of also boosting convalescent individuals with mRNA vaccines.

\section{Keywords}

T cells, SARS-CoV-2, mRNA vaccination, COVID-19, Omicron, B.1.1.529

\section{Main}

Continuously emerging variants of concern (VOC) sustain the pandemic state of SARS-CoV2. Indeed, the current SARS-CoV-2 VOC omicron (B.1.1.529) is a global health care threat due to its substantially increased infectivity and efficient ability to evade neutralisation by spikespecific antibodies induced by previous infection or vaccination ${ }^{1-6}$. Indeed, an approx. 5 to 25fold higher concentration of neutralising antibodies is required to abolish infectivity of omicron compared to the delta VOC. Both, increased infectivity and evasion from the humoral response is at least partially based on mutations in the receptor binding domain (RBD) resulting in enhanced affinity to ACE2 receptor and disruption of antibody binding sites ${ }^{1}$. Multiple additional mutations in the spike protein of omicron confer the risk of a broad evasion from the immune response that was readily established through vaccination and previous infection. This assumption is in line with the reportedly higher risk of break through infection with omicron in convalescents and vaccinees with complete initial immunization. Severe disease after omicron infection, is, however, not common and may be prevented by broadly cross-reactive cellular immunity ${ }^{7}$. Indeed, an important role of vaccine- and infection-induced T cells in preventing 
severe COVID-19 has been reported prior to the emergence of omicron ${ }^{8-11}$. Currently, very little information is available about the evasion of omicron from cellular immunity although first data indicate that this occurs to a lesser extend compared to evasion from humoral immunity. The SARS-CoV-2-specific T cell epitope repertoire has been studied in some detail ${ }^{11-16}$ (reviewed in ${ }^{17}$ ), however, comparative in-depths studies of the epitope repertoire targeted by infection- versus vaccine-induced $T$ cell responses are so far lacking, hindering the precise prediction of the immune escape potential of emerging VOC including omicron from the T cell response in convalescents compared to vaccinees.

To address this important issue, we studied SARS-CoV-2-specific $\mathrm{T}$ cell responses in convalescents recovered from natural SARS-CoV-2 infection $(n=19)$ as well as individuals after two $(n=16)$ and three $(n=7)$ doses of SARS-CoV-2 vaccination (Pfizer/BioNTech mRNA vaccine) (Supplementary Table 1). We first mapped the overall SARS-CoV-2-specific CD8+ $T$ cell response and tested a set of 43 previously described immunodominant SARS-CoV-2specific CD8+ T cell epitopes restricted by common HLA class I alleles ${ }^{11-16}$ in epitope-specific $\mathrm{T}$ cell cultures followed by cytokine staining (intracellular interferon-gamma production). Convalescents displayed CD8+ $T$ cell responses against the majority of epitopes that were distributed over all viral proteins, with spike-specific epitopes definitively not being dominant (Figure 1A, left column). In vaccinees, in contrast and as expected, CD8+ T cell responses were predominantly directed against spike epitopes (Figure 1A, right column). Few CD8+ $T$ cell responses targeted non-spike epitopes, with the $\mathrm{HLA}-\mathrm{B}^{*} 07 / \mathrm{N}_{105-113}$ epitope being the main target. For this epitope, cross-recognition by $T$ cells against common cold corona viruses has been previously suggested ${ }^{18-20}$. Importantly, individual spike-specific CD8+ T cell epitopes were more often targeted in vaccinees compared to convalescents, and the spike-specific CD8+ $T$ cell repertoire also appeared to be broader in vaccinees compared to convalescents. When we compared the corresponding viral sequences between ancestral and omicron SARSCoV-2 variants, only a single tested optimal CD8+ T cell epitope was affected by viral variation (Figure 1A, shown in red).

To further analyze the striking differences in spike-specific CD8+ $T$ cell responses in convalescents versus vaccinees and the nearly complete absence of viral variations in the targeted epitopes in more detail, we analysed these responses using overlapping peptides spanning the whole spike protein (180 18-mer peptides, sliding by 7 amino acids and thus overlapping by 11 amino acids). For all positive responses, we evaluated the overlapping peptide for described optimal epitopes restricted by the HLA class I alleles expressed by the respective individual. If no matching optimal epitopes has been previously described, we performed an in silico analysis to predict the most likely HLA class I restriction and optimal epitope. Using this comprehensive approach, we identified an overall substantially broader repertoire of spike-specific CD8+ $\mathrm{T}$ cell responses in vaccinees (Fig. 1B, lower panel, and 
112 Supplementary Figure 1A) compared to convalescents (Fig. 1B, upper panel and

113 Supplementary Figure 1B). Indeed, in convalescents, no HLA class I allele restricted more

114 than two spike-specific CD8+ T cell epitopes, while several HLA class I alleles restricted five or more spike-specific CD8+ T cell epitopes in vaccinees. In addition, we detected more spikespecific CD8+ $T$ cell responses per individual in vaccinees compared to convalescents (Fig. 1C). Hence, the increased breadth of the spike-specific CD8+ T cell response in vaccinees was evident on an individual and on a population level. To test whether an increased breadth of the spike-specific response in vaccinees is also evident for CD4+ $T$ cells, we analysed the CD4+ $T$ cell response applying the overlapping peptides spanning the whole spike protein as described above. In contrast to the CD8+ T cell response, the spike-specific CD4+ $T$ cell response showed a more limited repertoire of targeted epitopes after vaccination compared to natural infection (Fig. 1D and Supplementary Figure 1). In particular, fewer spike-specific CD4+ T cell epitopes were restricted by single HLA class II alleles (Fig. 1D) and fewer CD4+ T cell responses were detectable per individual (Fig. 1E) in vaccinees compared to convalescents. The spike-specific CD4+ T cell repertoire was therefore limited with respect to the individual and the population-based CD4+ $T$ cell response in vaccinees. Still, the fewer targeted spike-specific CD4+ T cell epitopes in vaccinees exhibited high conservation between ancestral and omicron SARS-CoV-2 as it is also the case for the majority of targeted epitopes in convalescents (Fig. 1D, variant epitopes shown in red). Comparing CD8+ and CD4+ T cell response (Fig. 1F), mRNA vaccination appears to particularly broaden and thus increase a cross-reactive spike-specific CD8+ T cell response.

134 Next, to assess the effect of boosting vaccination- or infection-induced $T$ cell responses by mRNA vaccination on the spike-specific CD8+ T cell repertoire, we performed the very same approach as described above using overlapping spike peptides to map spike-specific CD8+ and CD4+ T cell responses in longitudinally followed vaccinees getting their $3^{\text {rd }}$ vaccine dose (Pfizer/BioNTech mRNA vaccine; $n=7$; Supplementary Table 1) and convalescent individuals that received an mRNA booster vaccination ( $n=3$; Supplementary Table 1). After the third mRNA vaccination, we observed a similarly broad and cross-reactive spike-specific CD8+ T cell repertoire and similarly limited but still cross-reactive spike-specific CD4+ T cell repertoire compared to the completed initial immunization with two vaccine doses (Fig. 2A and Supplementary Fig. 2A). Strikingly, however, we detected CD8+ T cell responses targeting more overlapping peptides after the mRNA boost vaccination in convalescents, representing a broader spike-specific CD8+ $T$ cell repertoire (Fig. 2B and Supplementary Fig. 2B). In contrast, the CD4+ $\mathrm{T}$ cell repertoire was similar before and after boost vaccination of convalescents (Fig. 2B and Supplementary Fig. 2B). Again, the identified CD8+ and CD4+ 
vaccination increased SARS-CoV-2-specific CD8+ $T$ cell responses targeting conserved regions within the spike protein of omicron in convalescent individuals. To address the question whether the observed broader spike-specific CD8+ $T$ cell repertoire after mRNA vaccination may also be beneficial for potentially emerging future SARS-CoV-2 VOC beyond omicron, we analyzed the $T$ cell response targeting highly conserved selective sweep regions in SARS-CoV- ${ }^{21}$ in convalescents versus vaccinees. Selective sweep regions mediate per definition an evolutionary advantage and therefore it is very likely that newly emerging SARS-CoV-2 VOC also harbor high conservation within these regions. Four different selective sweep regions have so far been described in the spike protein of SARS-CoV- $2^{21}$ that also exhibit, as expected, a high degree of amino acid homology among the already evolved SARS-CoV-2 VOC (Fig. 3A/B). Importantly, compared to convalescents more vaccinated individuals showed spike-specific CD8+ T cell responses targeting epitopes within the highly conserved selective sweep regions indicating a spike-specific CD8+ T cell response with focussed targeting of highly conserved regions after vaccination (Fig. 3C). A similarly focussed spike-specific CD4+ T cell response was not evident after vaccination (Fig. 3D). Hence, a broadly cross-recognizing spike-specific CD8+ $T$ cell response is induced after mRNA vaccination that may be also reactive towards emerging SARS-CoV-2 VOC in future beyond omicron.

In conclusion, our data indicate that (1) convalescents target a variety of SARS-CoV-2-specific CD8+ T cell epitopes over the complete SARS-CoV-2 proteome with spike-specific CD8+ T cell responses being not dominant; (2) in contrast to the CD4+ T cell response, CD8+ T cell responses in vaccinees are focussed on a broader repertoire of highly conserved spikespecific CD8+ T cell epitopes leading to an increased cross-recognizing potential; (3) boosting convalescents with mRNA vaccination results in a broader spike-specific CD8+ $\mathrm{T}$ cell response; and (4) CD8+ and CD4+ T cell responses in both, convalescents as well as vaccinees, target epitopes that are highly conserved between ancestral, omicron and potentially future emerging SARS-CoV-2 variants. Hence, our data emphasize the relevance of $\mathrm{mRNA}$ vaccine-induced spike-specific CD8+ T cell responses in combating emerging SARSCoV-2 VOC including omicron and support the benefit of also boosting convalescent individuals with mRNA vaccines.

\section{Data availability}

182 All requests for raw and analyzed data and materials are promptly reviewed by the University 183 of Freiburg Center for Technology Transfer to verify if the request is subject to any intellectual 184 property or confidentiality obligations. Patient-related data not included in the paper were 
generated as part of clinical examination and may be subject to patient confidentiality. Any data and materials that can be shared will be released via a Material Transfer Agreement.

\section{Acknowledgements}

We thank all donors for participating in the current study and FREEZE-biobank-Center for biobanking of the Freiburg University Medical Center and the Medical Faculty for support. This study was supported by grants from the German Federal Ministry of Education and Research (01KI2077 to G.K., M.S., M.H., and R.T.) and the German Research Foundation (272983813 to B.B., T.B., R.T., M.H., and C.N.-H.; 256073931 to B.B., R.T., M.H., and C.N.-H.; 413517907 to H.L.). This work was also supported by the project "Virological and immunological determinants of COVID-19 pathogenesis - lessons to get prepared for future pandemics (KA1Co-02 "COVIPA")", a grant from the Helmholtz Association's Initiative and Networking Fund (to R.T. and M.H.). M.H. is furthermore supported by the Margarete von Wrangell fellowship (State of Baden-Wuerttemberg).

\section{Author contributions}

J.L.-M., H.L., K.W., V.K. and V.O. planned, performed and analyzed experiments with the help of E.S.A., A.G. and M.R.. J.L.-M., H.L., K.W., V.K. and V.O. contributed equally to this work. H.L., N.R., V.G., D.A., S.R., T.W., D.S. and C.F.W. were responsible for donor recruitment. F.E. performed four-digit HLA-typing by next generation sequencing. S.G., K.C., M.S. and G.K. provided virological expertise. B.B. and T.B. contributed to data interpretation. T.B. supervised the CD4+ T cell analysis and interpreted data. R.T., M.H. and C.N.-H. designed the study and contributed to experimental design and planning. J.L.-M., H.L., V.O., R.T., M.H. and C.N.H. interpreted data and wrote the manuscript. T.B., C.N.H., M.H. and R.T. are shared last authors.

\section{Declaration of interest}

The authors have nothing to declare.

\section{References}

1. Cameroni E, Bowen JE, Rosen LE, et al. Broadly neutralizing antibodies overcome SARS-CoV-2 Omicron antigenic shift. Nature 2021.

2. Dejnirattisai W, Huo J, Zhou D, et al. SARS-CoV-2 Omicron-B.1.1.529 leads to widespread escape from neutralizing antibody responses. Cell 2022;Online JAN 042022 (DOI 10.1016/j.cell.2021.12.046).

3. Garcia-Beltran WF, St Denis KJ, Hoelzemer A, et al. mRNA-based COVID-19 vaccine boosters induce neutralizing immunity against SARS-CoV-2 Omicron variant. Cell 2022.

4. Hoffmann M, Krüger N, Schulz S, et al. The Omicron variant is highly resistant against antibodymediated neutralization - implications for control of the COVID-19 pandemic. Cell 2021;Online DEC 23 2021 (DOI 10.1016/j.cell.2021.12.032). 
5. Nemet I, Kliker L, Lustig $\mathrm{Y}$, et al. Third BNT162b2 vaccination neutralization of SARS-CoV-2 omicron infection. N Engl J Med 2021;Online DEC 29, 2021 (DOI: 10.1056/NEJMc2119358).

6. Schmidt F, Muecksch F, Weisblum $Y$, et al. Plasma neutralization of the SARS-CoV-2 omicron variant. N Engl J Med 2021;Online DEC 30, 2021 (DOI: 10.1056/NEJMc2119641).

7. Collie S, Champion J, Moultrie H, Bekker LG, Gray G. Effectiveness of BNT162b2 Vaccine against Omicron Variant in South Africa. N Engl J Med 2021.

8. Grifoni A, Weiskopf D, Ramirez SI, et al. Targets of T Cell Responses to SARS-CoV-2 Coronavirus in Humans with COVID-19 Disease and Unexposed Individuals. Cell 2020;181:1489-501 e15.

9. Oberhardt $\mathrm{V}$, Luxenburger $\mathrm{H}$, Kemming J, et al. Rapid and stable mobilization of CD8(+) T cells by SARS-CoV-2 mRNA vaccine. Nature 2021;597:268-73.

10. Painter MM, Mathew D, Goel RR, et al. Rapid induction of antigen-specific CD4(+) T cells is associated with coordinated humoral and cellular immunity to SARS-CoV-2 mRNA vaccination. Immunity 2021;54:2133-42 e3.

11. Schulien I, Kemming J, Oberhardt V, et al. Characterization of pre-existing and induced SARSCoV-2-specific CD8(+) T cells. Nat Med 2021;27:78-85.

12. Ferretti AP, Kula T, Wang Y, et al. Unbiased Screens Show CD8(+) T Cells of COVID-19 Patients Recognize Shared Epitopes in SARS-CoV-2 that Largely Reside outside the Spike Protein. Immunity 2020;53:1095-107 e3.

13. Hu C, Shen $M$, Han $X$, et al. Identification of cross-reactive CD8(+) T cell receptors with high functional avidity to a SARS-CoV-2 immunodominant epitope and its natural mutant variants. Genes Dis 2022;9:216-29.

14. Kared H, Redd AD, Bloch EM, et al. SARS-CoV-2-specific CD8+ T cell responses in convalescent COVID-19 individuals. J Clin Invest 2021;131.

15. Nelde A, Bilich T, Heitmann JS, et al. SARS-CoV-2-derived peptides define heterologous and COVID-19-induced T cell recognition. Nat Immunol 2021;22:74-85.

16. Saini SK, Hersby DS, Tamhane T, et al. SARS-CoV-2 genome-wide T cell epitope mapping reveals immunodominance and substantial CD8(+) T cell activation in COVID-19 patients. Sci Immunol 2021;6. 17. Grifoni A, Sidney J, Vita R, et al. SARS-CoV-2 human T cell epitopes: Adaptive immune response against COVID-19. Cell Host Microbe 2021;29:1076-92.

18. Lineburg KE, Grant EJ, Swaminathan S, et al. CD8(+) T cells specific for an immunodominant SARS-CoV-2 nucleocapsid epitope cross-react with selective seasonal coronaviruses. Immunity 2021;54:1055-65.e5.

19. Nguyen THO, Rowntree LC, Petersen J, et al. CD8(+) T cells specific for an immunodominant SARS-CoV-2 nucleocapsid epitope display high naive precursor frequency and TCR promiscuity. Immunity 2021;54:1066-82.e5.

20. Peng Y, Felce SL, Dong D, et al. An immunodominant NP(105-113)-B*07:02 cytotoxic $T$ cell response controls viral replication and is associated with less severe COVID-19 disease. Nat Immunol 2022;23:50-61.

21. Kang L, He G, Sharp AK, et al. A selective sweep in the Spike gene has driven SARS-CoV-2 human adaptation. Cell 2021;184:4392-400 e4. 


\section{Online methods}

\section{Ethics}

Patients were recruited at the Freiburg University Medical Center, Germany between August 2019 and January 2022. Written informed consent was obtained from all participants. The study was conducted according to federal guidelines and local ethics committee regulations (Albert-Ludwigs-University Freiburg, Germany; vote: \#21-1135 and \#21-1372) and the Declaration of Helsinki (1975).

\section{Study Cohort \& Clinical definitions}

19 convalescent individuals following a mild course of SARS-CoV-2 infection were analyzed. All patients were confirmed to have a test positive for SARS-CoV-2 using PCR with reverse transcription from an upper respiratory tract (nose and throat) swab tested at an accredited laboratory. The degree of severity was identified according to recommendations from the World Health Organization. Moreover, 16 individuals were screened 2-4 weeks after first mRNA boost vaccination (Pfizer/BioNTech BNT162) and 7 of the same individuals 2-4 weeks after second boost vaccination (Pfizer/BioNTech BNT162). Three individuals were analyzed who had a mild course of SARS-CoV-2 infection and were vaccinated once with mRNA vaccine (Pfizer/BioNTech BNT162).

\section{PBMC isolation}

Peripheral blood mononuclear cells (PBMCs) were isolated from anticoagulated blood samples with density gradient centrifugation (Pancoll separation medium, PAN Biotech GmbH; Aidenbach, Germany). Subsequently stored at $-80^{\circ} \mathrm{C}$ resuspended in RPMI 1640 medium supplemented with $10 \%$ fetal calf serum, $1 \%$ penicillin/streptomycin, and $1.5 \%$ HEPES buffer $1 \mathrm{~mol} / \mathrm{L}$ (complete medium; all additives from Thermo Scientific (Waltham, MA)) until further usage.

\section{Peptides}

A total of 182 overlapping peptides that spanned the SARS-CoV-2 spike sequence (Gene Bank Accession code MN908947.3) were synthesized as 18-mers overlapping by 11 amino acids with a free amine $\mathrm{NH} 2$ terminus and a free acid $\mathrm{COOH}$ terminus with standard Fmoc chemistry and a purity of $>70 \%$ (Genaxxon Bioscience). Similarly, 60 predescribed SARSCoV-2 specific optimal CD8 ${ }^{+} \mathrm{T}$ cell epitopes were synthesized.

In vitro expansion and intracellular IFNY staining with overlapping peptides or optimal predescribed $C D 8^{+} T$ cell epitopes 
In vitro expansion with OLPs or optimal epitopes was performed as follows: $20 \%$ of the PBMCs were stimulated with a pool of all 181 SARS-CoV-2 spike OLPs or optimal epitopes (10 $\mu \mathrm{g}$ $\mathrm{ml}^{-1}$ ) for $1 \mathrm{~h}$ at $37^{\circ} \mathrm{C}$, washed and co-cultured with the remaining PBMCs in RPMI medium supplemented $20 \mathrm{U} \mathrm{ml-1}$ with recombinant IL-2. On day 10, intracellular IFNy staining was performed with pooled OLPs (45 pools with 4 OLP each). Therefore, cells were re-stimulated with OLP pools $(50 \mu \mathrm{M})$, DMSO as negative control or PMA and ionomycin as positive control in the presence of brefeldin A an IL-2. After $5 \mathrm{~h}$ of incubation at $37^{\circ} \mathrm{C}$, cells were stained for surface markers (CD8+, CD4+; Viaprobe) and intracellular markers (IFNY). Subsequently, on day 12-14 the single overlapping peptides of positive pools and HLA-matched optimal CD8 ${ }^{+} \mathrm{T}$ cell epitopes were tested by intracellular cytokine staining. Viral amino acid sequences of positive individual OLPs were analysed for pre-described minimal epitopes ${ }^{1-6}$ or the best HLAmatched predicted candidate using the Immune Epitope Database website (using two prediction algorithms ANN 4.0 and NetMHCpan EL 4.123 for 8-mer, 9-mer and 10-mer peptides with half-maximal inhibitory concentration (IC50) of $<500 \mathrm{nM}$ ).

\section{Multiparametric flow cytometry}

The following antibodies were used or flow cytometry: Anti-CD8-APC (SK-1, 1:200), anti-CD4efluor450 (RPA-T4, 1:250), anti-IFN-Y-FITC (25723.11, 1:8), fixable Viability Dye (eFluor506 $1: 200,1: 400)$. After fixation of cells in $2 \%$ paraformaldehyde/PBS (Sigma; Germany), acquisition was performed on BD FACSCanto (BD; Germany). Data were analyzed with FlowJo, LLC (10.0.7r2, BD; USA).

\section{ELISA}

Spike-binding antibodies were assessed by Anti-SARS-CoV-2-QuantiVac-ELISA (IgG) (Euroimmun; Germany) detecting S1 lgG (<25.6 BAU/ml: negative; 25.6-35.1 BAU/ml: marginally positive; $\geq 35.2 \mathrm{BAU} / \mathrm{ml}$ : positive) according to manufacturer's instructions.

\section{Sequence alignment}

Sequence homology analyses were performed in Geneious ${ }^{\circledR} \quad 11.0 .5$ (https://www.geneious.com/) using Clustal Omega 1.2.2 alignment with default settings ${ }^{7-9}$. Reference genome of human SARS-CoV-2 (MN908947.3) was downloaded from NCBI database. SARS-CoV-2 epitopes were then mapped to the corresponding protein alignment. Selective sweep regions were indicated as described by Kang et al. ${ }^{10}$. SARS-CoV-2 variants of concern were identified via CoVariants (https://covariants.org/). 
74 1. Grifoni A, Sidney J, Zhang Y, Scheuermann RH, Peters B, Sette A. A Sequence Homology and 75 Bioinformatic Approach Can Predict Candidate Targets for Immune Responses to SARS-CoV-2. Cell Host 76 Microbe 2020;27:671-80 e2.

77 2. Kared H, Redd AD, Bloch EM, et al. SARS-CoV-2-specific CD8+ T cell responses in convalescent 78 COVID-19 individuals. J Clin Invest 2021;131.

$793 . \quad$ Saini SK, Hersby DS, Tamhane T, et al. SARS-CoV-2 genome-wide T cell epitope mapping reveals 80 immunodominance and substantial CD8(+) T cell activation in COVID-19 patients. Sci Immunol 2021;6. 81 4. Schulien I, Kemming J, Oberhardt V, et al. Characterization of pre-existing and induced SARS82 CoV-2-specific CD8(+) T cells. Nat Med 2021;27:78-85.

83 5. Shomuradova AS, Vagida MS, Sheetikov SA, et al. SARS-CoV-2 Epitopes Are Recognized by a 84 Public and Diverse Repertoire of Human T Cell Receptors. Immunity 2020;53:1245-57 e5.

85 6. Tarke A, Sidney J, Kidd CK, et al. Comprehensive analysis of T cell immunodominance and 86 immunoprevalence of SARS-CoV-2 epitopes in COVID-19 cases. Cell Rep Med 2021;2:100204.

87 7. Sievers F, Barton GJ, Higgins DG. Multiple sequence alignment. Bioinformatics 2020;227:2278850

89 8. Sievers F, Higgins DG. Clustal Omega for making accurate alignments of many protein 90 sequences. Protein Sci 2018;27:135-45.

91 9. Sievers $F$, Wilm A, Dineen $D$, et al. Fast, scalable generation of high-quality protein multiple 92 sequence alignments using Clustal Omega. Mol Syst Biol 2011;7:539.

93 10. Kang L, He G, Sharp AK, et al. A selective sweep in the Spike gene has driven SARS-CoV-2 human 94 adaptation. Cell 2021;184:4392-400 e4. 
A

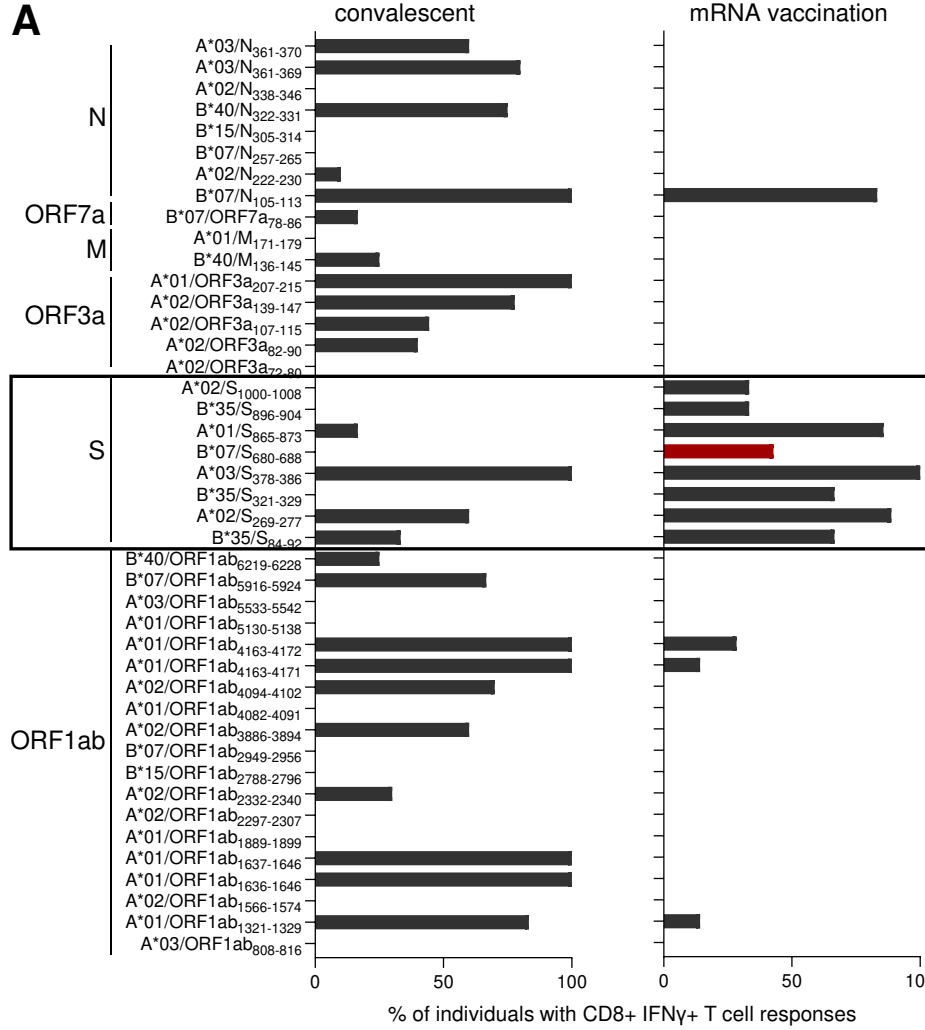

C convalescent $(n=19)$
mRNA vaccination $(\mathrm{n}=16)$

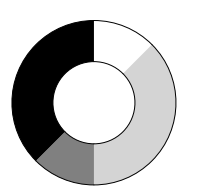

mRNA vaccination $(n=16)$

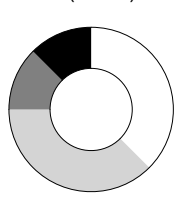

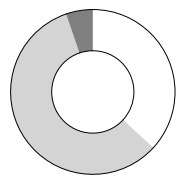

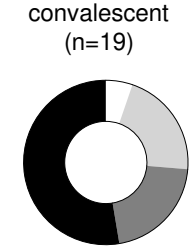

$\mathrm{n}^{\circ}$ of $\mathrm{CD} 8+\mathrm{T}$ cell responses per individual

$$
\begin{aligned}
& \text { - None } \\
& =1-2 \\
& =3-5 \\
& ->5
\end{aligned}
$$

$$
\begin{gathered}
\frac{\mathrm{n}^{\circ} \text { of } C D 4+T \text { cell }}{\text { responses per individual }} \\
\square \text { None } \\
\square 1-2 \\
=3-5 \\
->5
\end{gathered}
$$

$\mathbf{F}$

F

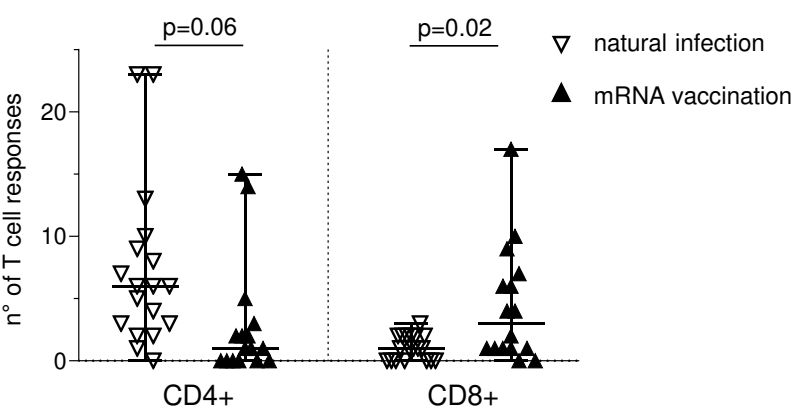

B
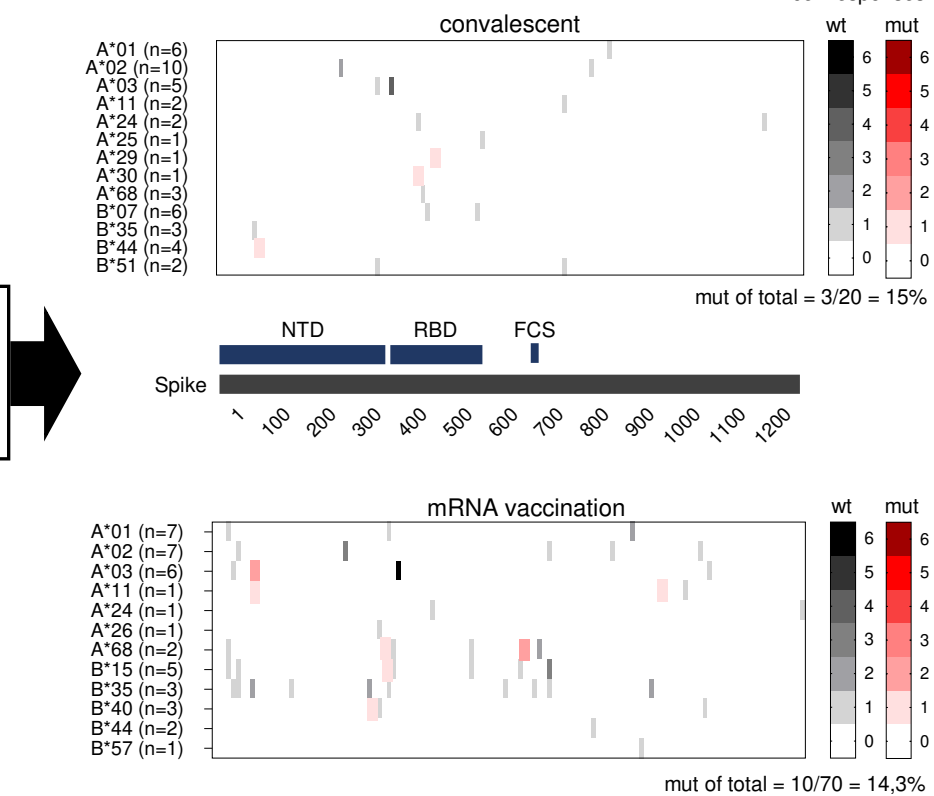

D

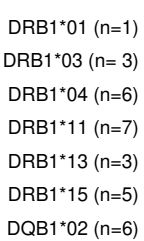
$\mathrm{DQB} 1{ }^{*} 02(\mathrm{n}=6)$

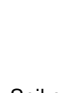
Spike
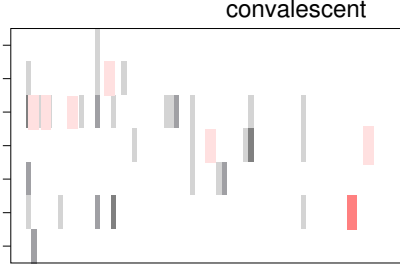

of CD4+ $\mathrm{T}$ cell responses

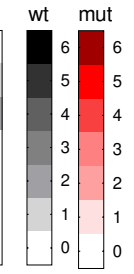

mut of total $=10 / 69=14,4 \%$
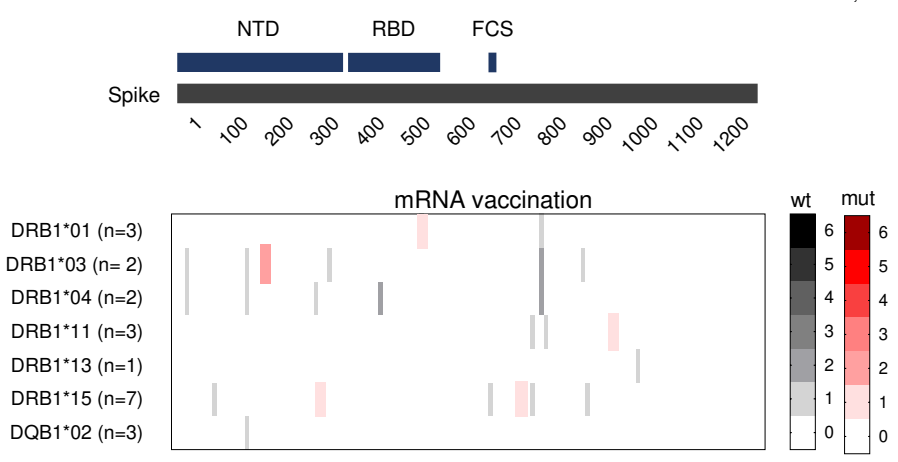

mut of total $=6 / 29=20,6 \%$

Figure 1: $C D 8+$ and $C D 4+T$ cell responses targeting conserved and mutated epitopes in omicron.

Percentages of CD8+ T cell responses to previously described optimal CD8+ T cell epitopes in ancestral SARS-CoV-2 protein (A). Number, location and percentages of spike-specific CD8+ (B) and CD4+ (D) T cell responses to overlapping peptides (OLP) that are detectable in SARS-CoV-2 convalescents and vaccinees who received two doses of Pfizer/BioNTech mRNA vaccine are depicted. Number of CD8+ (C,F) and CD4+ $(E, F)$ T cell responses targeting spike-specific epitopes per individual in convalescents $(n=19)$ and vaccinees $(n=16)$. mean and range are depicted, statistical analysis was performed with paired t test. 


\section{A}
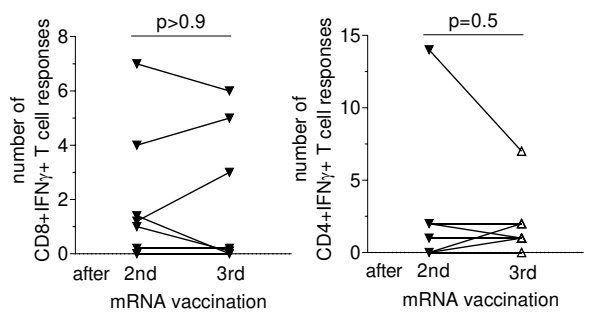

exemplary individual after $2^{\text {nd }}$ and $3^{\text {rd }} \mathrm{mRNA}$ vaccination:
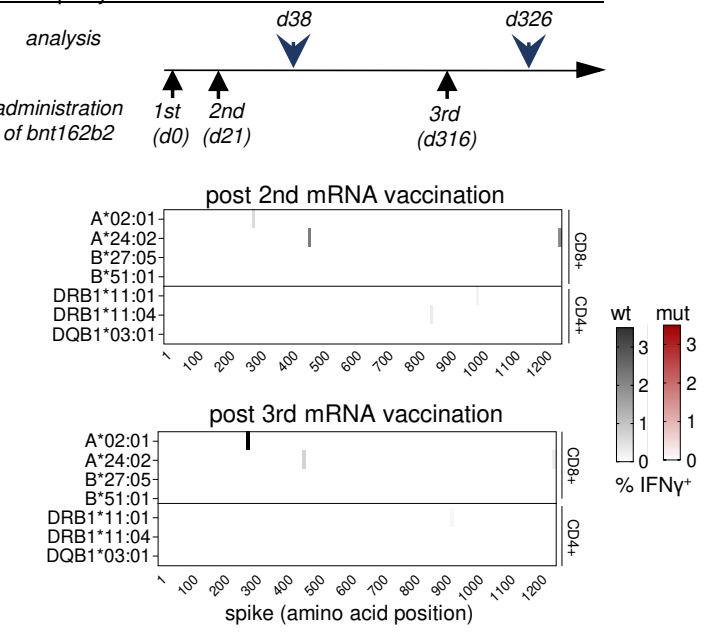

B

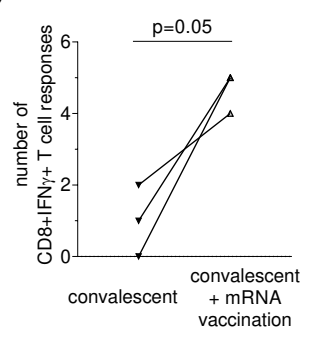

exemplary individual after natural infection and vaccination:
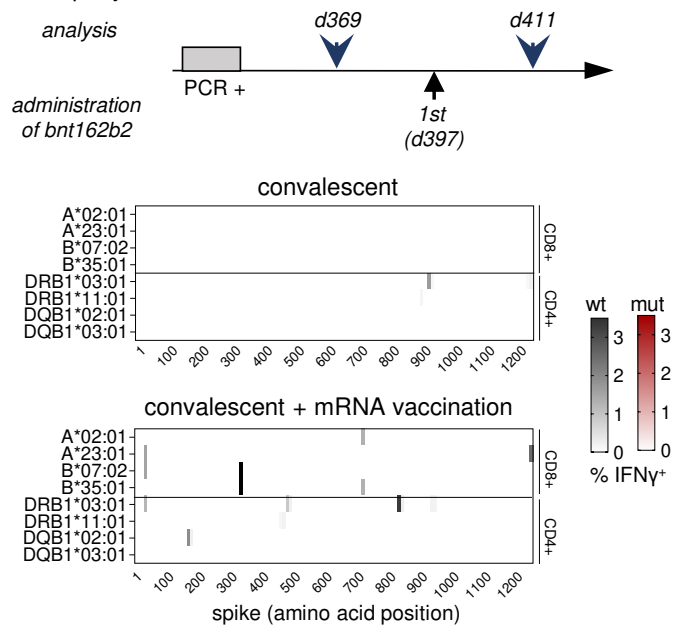

Figure 2: Boosted vaccine- and infection-induced spike-specific CD8+ and CD4+ T cell responses.

Number, location and percentages of spike-specific CD8+ and CD4+ T cell responses to overlapping peptides (OLP) that are detectable in SARS-CoV-2 vaccinees after the $2^{\text {nd }}$ versus after the $3^{\text {rd }}$ dose $(A)$ of Pfizer/BioNTech mRNA vaccine (measured 2-4 weeks after vaccination) and in SARS-CoV-2 convalescents who subsequently received a single dose (B) of Pfizer/BioNTech mRNA boost vaccination (measured 2 weeks after vaccination) are depicted. Targeted epitopes with sequence variations in omicron are marked in red. statistical analysis was performed with paired $t$ test. 


\section{A}

RBD

FCS

700

800

900

$1,0,00$

$1,1.00$

$1,200 \quad 1,274$

B

selective sweep 1

Alpha

Beta

Gamma

Delta

Omicron

TESIVRFPNITNLCPFGEVFNATRFASVYAWNRKRISNCVADYSVLYNSASFSTFKCYGVSPTKLNDLCFTNVYADSFVIRGDEVRQIAPGQTGKIADYNYKLPDDFTGCVI

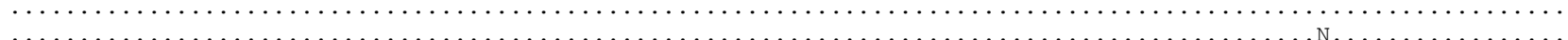

.

$\ldots \ldots \ldots \ldots \ldots$

..........

selective sweep 2

B1 VCGPKKSTNLVKNKCVNFNFNG

Alpha

Beta

Gamma

Delta

$\ldots \ldots \ldots \ldots \ldots \ldots$

$\ldots \ldots \ldots \ldots \ldots \ldots$

$\ldots \ldots \ldots \ldots \ldots \ldots$

$\ldots \ldots \ldots \ldots \ldots \ldots$

selective sweep 3

Omicron

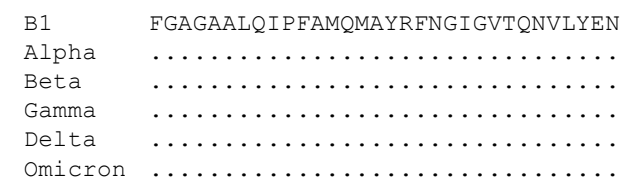

selective sweep 4

B1 QLSSNFGAISSVLNDILSRLDKVEAEVQIDRLITGRLQSLQTYVTQQLIRAAEIRASANLAATKMSECVLGQSKRVDFCGKGYHLM

\section{Alpha}

Beta

Gamma

Delta

Omicron

C

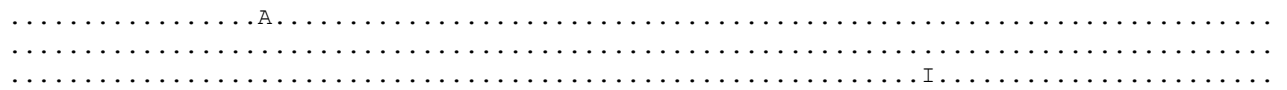

.

convalescent

$(n=19)$

mRNA vaccination $(n=16)$
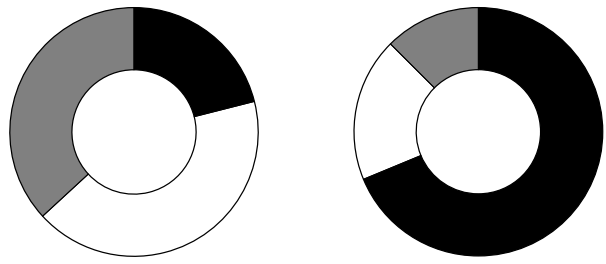

1 or more $\mathrm{CD}^{+} \mathrm{T}$ cell response(s) within selective sweep $\mathrm{CD}^{+} \mathrm{T}$ cell response only outside selective sweep no $\mathrm{CD}^{+} \mathrm{T}$ cell responses
D convalescent $(n=19)$

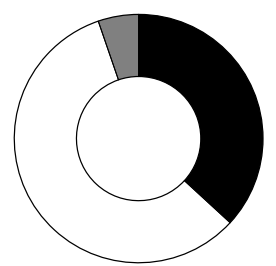

1 or more CD4+ T cell response(s) within selective sweep CD4+ T cell response only outside selective sweep no CD4+ T cell responses

\section{Figure 3: Spike-specific CD8+ and CD4+ T cell responses targeting selective sweep regions.}

Schematic representation of four selective sweep regions (SS1-4) in the spike protein of SARS-CoV-2 (A). Amino acid sequences of the selective sweep regions 1-4 in the spike protein of ancestral SARS-CoV-2 and VOC alpha, beta, gamma, delta and omicron (B). Vaccinees and convalescents with CD8+ (C) and CD4+ (D) T cell responses within and outside selective sweep regions 1-4. 
exemplary individual after $2^{\text {nd }}$ and $3^{\text {rd }}$ mRNA vaccination:

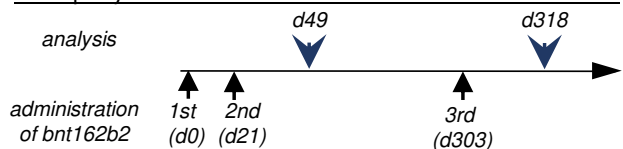

post 2nd mRNA vaccination
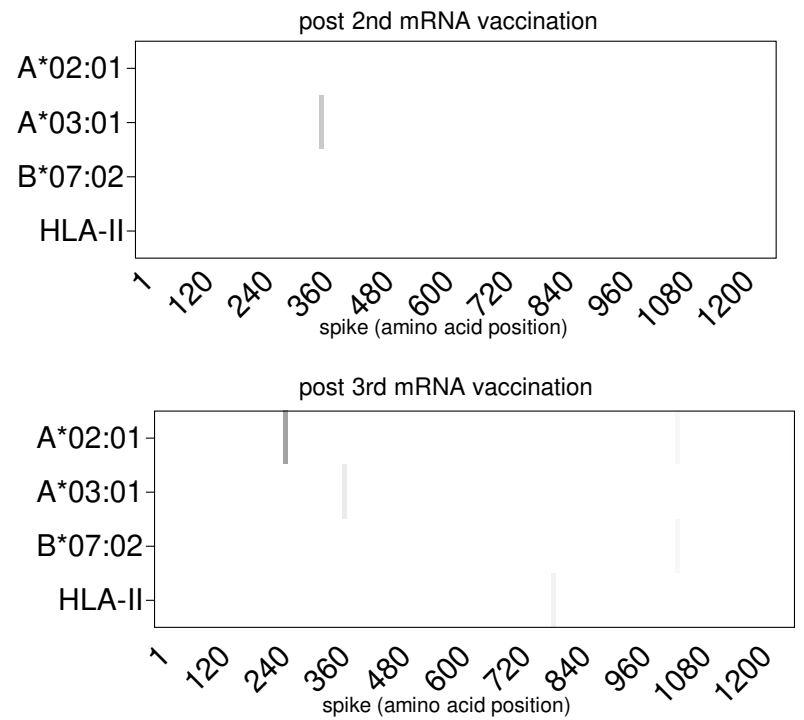

B

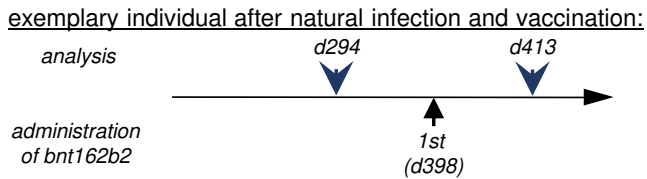

convalescent

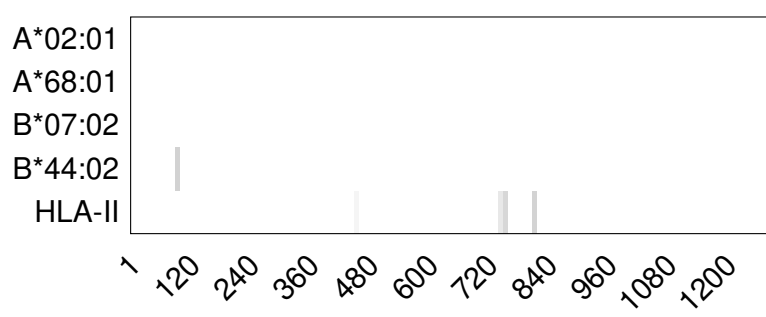

Convalescent + mRNA vaccination

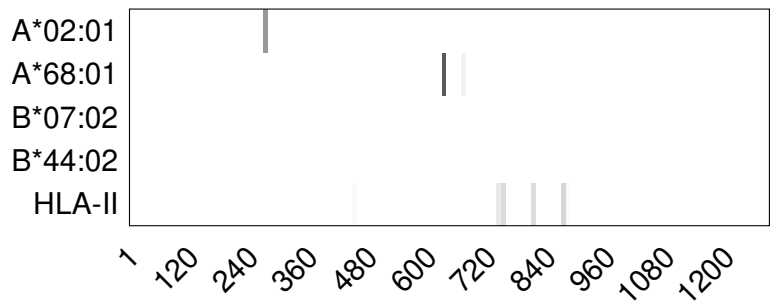

exemplary individual after $2^{\text {nd }}$ and $3^{\text {rd }}$ mRNA vaccination:
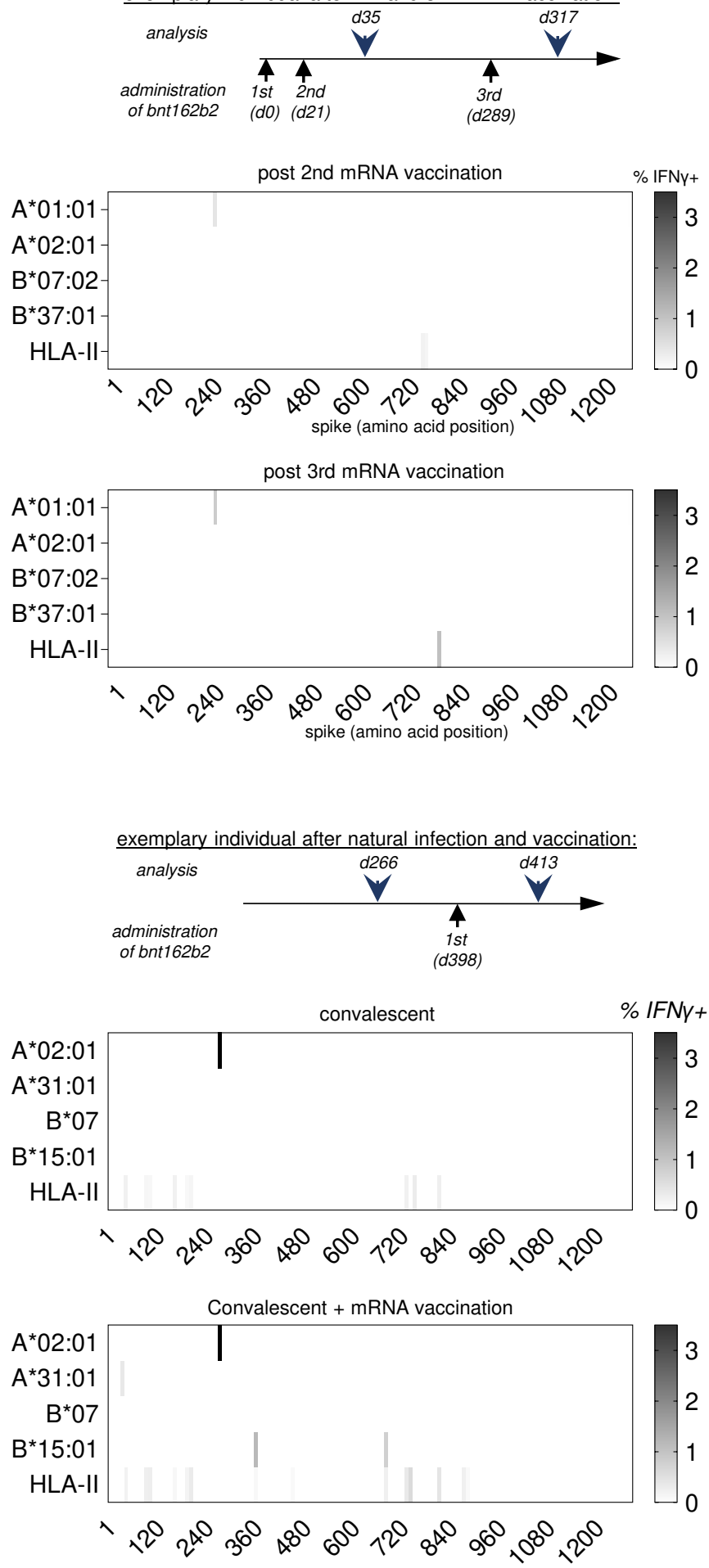

Extended Data 2: Boosted vaccine- and infection-induced spike-specific CD8+ and CD4+ T cell responses in exemplary individuals.

Number and location of spike-specific CD8+ and CD4+ T cell responses to overlapping peptides (OLP) that are detectable in exemplary SARS-CoV-2 vaccinees after the $2^{\text {nd }}$ versus after the $3^{\text {rd }}$ dose $(A)$ of Pfizer/BioNTech mRNA vaccine (bnt162b2) and in exemplary SARS-CoV-2 convalescents who subsequently received a single dose (B) of Pfizer/BioNTech mRNA boost vaccination are depicted. 


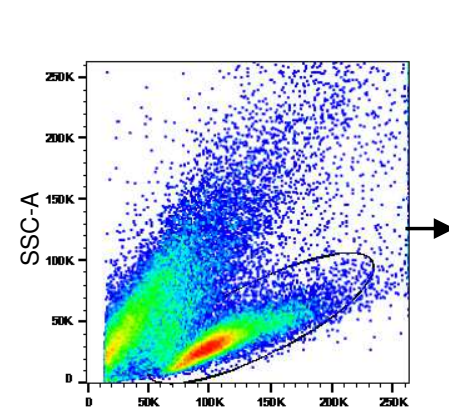

FCS-A

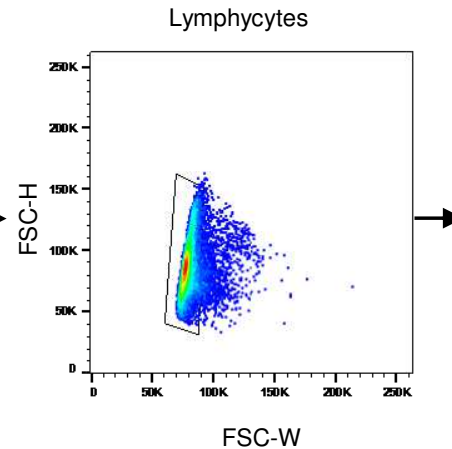

FSC-W

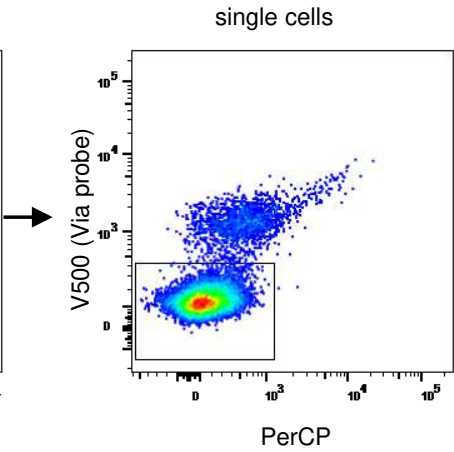

PerCP

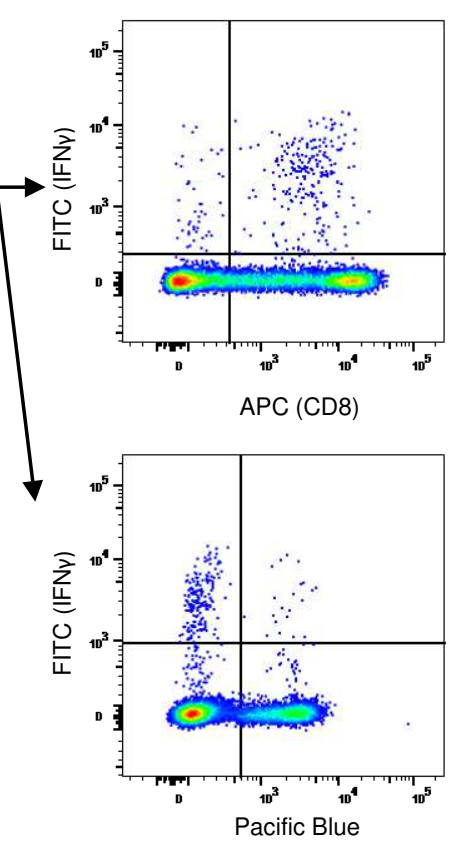

\section{Extended Data 3: Gating strategy}

Lymphocytes were gated on FSC-A and SSC-A, Doublet exclusion on FSC-H and FSC-W, Exclusion of dead cells, Gating on CD8+ or CD4+ cells. 


\begin{tabular}{|c|c|c|c|c|c|c|c|c|c|c|c|c|}
\hline \multirow{2}{*}{ Donor ID } & \multirow{2}{*}{ sex } & \multirow{2}{*}{ Age } & \multirow{2}{*}{ HLA class I } & \multirow{2}{*}{ HLA class II } & \multirow{2}{*}{ cohort } & \multirow{2}{*}{$\begin{array}{l}\text { Vaccine } \\
\text { 1. dose }\end{array}$} & \multirow{2}{*}{$\begin{array}{l}\text { Vaccine } \\
\text { 2. dose }\end{array}$} & \multirow{2}{*}{$\begin{array}{l}\text { Vaccine } \\
\text { 3. dose }\end{array}$} & \multirow{2}{*}{$\begin{array}{l}\text { Natural } \\
\text { infection }\end{array}$} & $\begin{array}{c}\text { Serum } \$ 1 \text { lgG levels } \\
\text { (BAU/m) after ratural } \\
\text { infection }\end{array}$ & $\begin{array}{l}\text { Serum S1 lgG1 levels } \\
\text { (BAU/ml) after 2. dose }\end{array}$ & $\begin{array}{l}\text { Serum } \mathrm{S} 1 \mathrm{ggG} 1 \text { levels } \\
\text { (BAU/m) after 3. dose }\end{array}$ \\
\hline & & & & & & & & & & \multicolumn{3}{|c|}{ 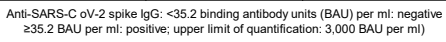 } \\
\hline 1 & $\mathrm{f}$ & 30 & $A^{*} 26: 01, B^{*} 07: 02, B^{*} 38: 01$ & 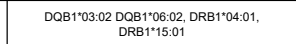 & vaccinated & BNT 16262 & BNT162b2 & BNT16262 & - & - & $>3000$ & - \\
\hline 2 & $\mathrm{~m}$ & 47 & $A^{*} 02: 01, A^{*} 24: 02, B^{*} 27: 05, B^{*} 51: 01$ & DQB1*03:01, DRB1*11:01, DRB1*11:04 & vaccinated & BNT162b2 & BNT162b2 & BNT162b2 & - & - & $>3000$ & . \\
\hline 3 & $\mathrm{~m}$ & 33 & $A^{*} 02: 01, A^{*} 03: 01, B^{*} 07: 02$ & DQB1*06:02, DRB1*15:01 & vaccinated & BNT162b2 & BNT162b2 & BNT162b2 & - & . & $>3000$ & . \\
\hline 4 & $\mathrm{~m}$ & 41 & $A^{*} 01: 01, A^{*} 02: 01, B^{*} 08: 01, B^{*} 40: 01$ & 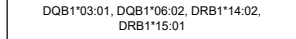 & vacinated & BNT162b2 & BNT162b2 & BNT162b2 & & - & 1522.56 & $>3000$ \\
\hline 5 & $\mathrm{f}$ & 37 & $A^{*} 02: 01, A^{*} 68: 01, B^{*} 15: 01, B^{*} 4: 01$ & DQB1*06:02 DQB1*06:03, DRB1*13:01, DRB1*15:01 & vaccinated & BNT162b2 & BNT162b2 & - & & - & $>3000$ & \\
\hline 6 & $\mathrm{~m}$ & 31 & $A^{*} 02: 01, A^{*} 03: 01, B^{*} 15: 01, B^{*} 57: 03$ & DQB 1*03:02, DQB 1*06:02, DRB 1*04:01, DRB 1*14:01 & vaccinated & BNT 16262 & BNT162b2 & . & - & & $>3000$ & . \\
\hline 7 & $\mathrm{~m}$ & 32 & $A^{*} 03: 01, A^{*} 03: 01, B^{*} 07: 02, B^{*} 44: 02$ & DQB1*03:01 DQB1*06:03, DRB1*11:198, DRB1*15:0 & vaccinated & BNT162b2 & BNT162b2 & - & - & - & $>3000$ & . \\
\hline 8 & $\mathrm{~m}$ & 43 & $A^{*} 03: 01, A^{*} 32: 01, B^{*} 07: 02, B^{*} 4: 02$ & DQB1*03:01, DQB $1 * 05: 03$, DRB**11:01, DRB**14:54 & vaccinated & BNT162b2 & $\mathrm{BNT} 162 \mathrm{~b} 2$ & - & & - & 1280.16 & \\
\hline 9 & $\mathrm{~m}$ & 60 & $A^{*} 02: 01, A^{*} 02: 01, B^{*} 08: 01, B^{*} 15: 01$ & DQB $1 * 02: 01$ DQB $1^{*} 03: 01, \mathrm{DRB}{ }^{*} \times 3: 01, \mathrm{DRB} 1^{*} 11: 01$ & vaccinated & BNT162b2 & BNT 162b2 & - & - & & $>3000$ & - \\
\hline 10 & f & 52 & $A^{*} 02: 01, A^{*} 68: 01, B^{*} 15: 01, B^{*} 44: 02$ & DQB $1 * 03: 01$ DQB $1 * 05: 03$, DRB1*11:03, DRB1*14:55 & vaccinated & BNT162b2 & BNT162b2 & BNT162b2 & - & - & $>3000$ & $>3000$ \\
\hline 11 & f & 35 & $A^{*} 01: 01, A^{*} 03: 01, B^{*} 07: 02, B^{*} 57: 01$ & DQB $1 * 03: 03, \mathrm{DQB}^{*}{ }^{* 06}: 02, \mathrm{DRB}{ }^{*} 07: 01, \mathrm{DRB} 1^{*} 15: 01$ & vaccinated & BNT162b2 & BNT162b2 & . & & - & $>3000$ & \\
\hline 12 & $\mathrm{f}$ & 44 & $A^{*} 01: 01, A^{*} 11: 01, B^{*} 15: 17, B^{*} 35: 01$ & DQB $1 * 05: 01$, DQB $1 * 06: 04$, DRB $1 * 01: 03$, DRB $^{1 * 13: 02}$ & vaccinated & BNT162b2 & BNT162b2 & . & - & . & $>3000$ & - \\
\hline 13 & f & 30 & $A^{*} 01: 01, A^{*} 02: 01, B^{*} 07: 02, B^{*} 37: 01$ & 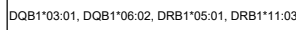 & vaccinated & BNT162b2 & BNT162b2 & $\mathrm{BNT} 162 \mathrm{~b} 2$ & - & & 1535.27 & $>3000$ \\
\hline 14 & $\mathrm{~m}$ & 30 & $A^{*} 0101, A^{*} 0201, B^{*} 1302, B^{*} 3502$ & DQB1*02:02, DQB1*03:01, DRB 1*07:01, DRB1*11:02 & vaccinated & BNT162b2 & BNT162b2 & . & - & - & $>3000$ & - \\
\hline 15 & $\mathrm{~m}$ & 35 & $A^{*} 01: 01, A^{*} 26: 01, B^{*} 07: 02, B^{*} 27: 05$ & 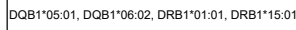 & vaccinated & BNT162b2 & BNT162b2 & BNT 162b2 & - & - & $>3000$ & 23000 \\
\hline 16 & $\mathrm{~m}$ & 41 & $A^{*} 01: 01, A^{*} 03: 01, B^{*} 08: 01, B^{*} 35: 01$ & DQB1*02:01, DQB1*05:01, DRB1*01:01, DRB1*03:01 & vaccinated & BNT162b2 & BNT $162 \mathrm{~b} 2$ & - & - & & $>3000$ & - \\
\hline 17 & $\mathrm{~m}$ & 57 & $A^{*} 02: 01, A^{*} 6: 01, B^{*} 07: 02, B^{*} 4: 02$ & 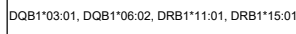 & $\begin{array}{c}\text { resolved } \\
\text { vaccinated }\end{array}$ & BNT16262 & - & - & mild & $>3000$ & $>3000$ (after 1 dose) & - \\
\hline 18 & $\mathrm{~m}$ & 55 & $A^{*} 02: 01, A^{*} 23: 01, B^{*} 07: 02, B^{*} 35: 01$ & 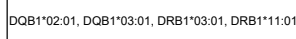 & $\begin{array}{c}\text { resolved } \\
\text { vaccinated }\end{array}$ & BNT162b2 & - & - & mild & 217.34 & $>3000$ (after 1 dose) & \\
\hline 19 & w & 56 & $A^{*} 02: 01, A^{*} 31: 01, B^{*} 07, B^{*} 15: 01$ & 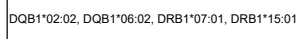 & $\begin{array}{c}\text { resolved } \\
\text { vaccinated }\end{array}$ & BNT 16262 & - & - & mild & - & - & - \\
\hline 20 & f & 28 & $A^{*} 11: 01, A^{*} 24: 02, B^{*} 51: 01$ & DQB1*03:03, DQB1*04:01, DRB1*04:01, DRB ${ }^{*} 09: 01$ & resolved & - & - & - & mild & 21.42 & - & - \\
\hline 21 & f & 31 & $\mathrm{~A}^{*} 02: 01, \mathrm{~B}^{*} 18: 01, \mathrm{~B}^{*} 38: 01$ & DQB1*02:02, DQB $1^{*} 06: 03, \mathrm{DRB} 1^{*} 07: 01, \mathrm{DRB} 1^{*} 13: 01$ & resolved & & . & . & mild & 46.69 & - & - \\
\hline 22 & $\mathrm{f}$ & 26 & $A^{*} 66: 01, A^{*} 68: 01, B^{*} 35: 01, B^{*} 44: 02$ & 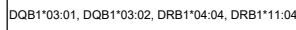 & resolved & - & - & . & mild & 46.69 & - & . \\
\hline 23 & $\mathrm{~m}$ & 37 & $A^{*} 02: 01, B^{*} 18: 01, B^{*} 39: 24$ & DQB1103:01, DRB1*11:01, DRB1*13:03 & resolved & - & . & . & mild & 104.29 & - & - \\
\hline 24 & f & 56 & $A^{*} 02: 01, A^{*} 03: 01, B^{*} 40: 12, B^{*} 51: 01$ & 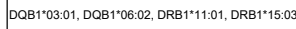 & resolved & . & - & . & mild & 136.91 & - & \\
\hline 25 & $\mathrm{~m}$ & 42 & $A^{*} 01: 01, B^{*} 50: 01$ & DQB1*02:02, DRB 1*07:01 & resolved & - & & . & mild & 40.32 & - & - \\
\hline 26 & f & 40 & $A^{*} 03: 01, A^{*} 11: 01, B^{*} 15: 01, B^{*} 35: 01$ & DQB1*03:01, DQB $1 * 06: 03$, DRB $1 * 04: 07$, DRB1*13:01 & resolved & - & . & . & mild & 77.91 & - & - \\
\hline 27 & $\mathrm{~m}$ & 52 & $A^{*} 01: 01, A^{*} 02: 01, B^{*} 37: 01, B^{*} 4: 02$ & 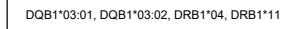 & resolved & - & - & . & mild & 151.47 & - & \\
\hline 28 & $\mathrm{~m}$ & 34 & $A^{*} 01: 01, A^{*} 03: 01, B^{*} 08: 01$ & DQB1*02:01, DRB ${ }^{*} 03: 01$ & resolved & - & & . & mild & 96.16 & . & . \\
\hline 29 & $\mathrm{~m}$ & 36 & $A^{*} 01: 01, A^{*} 03: 01, B^{*} 37: 01, B^{*} 40: 01$ & 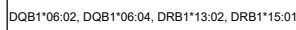 & resolved & . & . & . & mild & 7.41 & - & - \\
\hline 30 & f & 50 & $A^{*} 01: 01, A^{*} 02: 01, B^{*} 15: 01, B^{*} 39: 01$ & DQB ${ }^{* 03} 03: 02$, DQB $1 * 05: 03$, DRB $1 * 04$, DRB1*14:54 & resolved & - & - & - & mild & 368.19 & - & - \\
\hline 31 & f & 27 & $A^{*} 02: 01, A^{*} 25: 01, B^{*} 07: 02, B^{*} 15: 24$ & DQB1*05:03, DQB $1 * 06: 03$, DRB $^{*} 13: 01$, DRB $^{*} 1^{*} 14: 54$ & resolved & - & . & . & mild & 101.34 & - & - \\
\hline 32 & $\mathrm{~m}$ & 36 & $A^{*} 01: 01, A^{*} 29: 02, B^{*} 07: 02, B^{*} 40: 01$ & DQB1*03:01, DRB1*01:03, DRB 1*11:01 & resolved & - & . & . & mild & 13.64 & - & . \\
\hline 33 & $\mathrm{~m}$ & 26 & $A^{*} 02: 01, A^{*} 31: 01, B^{*} 07: 02, B^{*} 15: 01$ & DQB1 $104: 02, \mathrm{DQB}_{1}{ }^{* 06}: 02, \mathrm{DRB} 1^{*} 12: 01, \mathrm{DRB} 1^{*} 15: 01$ & resolved & - & & . & mild & 197.77 & . & . \\
\hline 34 & $\mathrm{~m}$ & 34 & $A^{*} 03: 01, A^{*} 24: 02, B^{*} 44: 03, B^{*} 45: 01$ & DQB1*03:01, DQB $1 * 06: 02$, DRB $1 * 04: 01$, DRB $^{*}{ }^{*} 15: 01$ & resolved & - & & . & mild & 33.97 & - & - \\
\hline 35 & $\mathrm{~m}$ & 31 & $\mathrm{~A} 30: 04, \mathrm{~A}^{*} 68: 01, \mathrm{~B}^{*} 40: 01, \mathrm{~B}^{*} 57: 03$ & DQB $1{ }^{*} 06: 02, \mathrm{DQB} 1{ }^{*} 06: 04, \mathrm{DRB} 1{ }^{\star} 13: 02, \mathrm{DRB} 1{ }^{*} 14: 54$ & resolved & - & & - & mild & 141.05 & - & - \\
\hline
\end{tabular}

